\title{
LỖI PHÁT ÂM CÁC ÂM XÁT /s/ VÀ / / / TIẾNG ANH CỦA SINH VIÊN CHUYÊN NGÀNH NGÔN NGỮ ANH TRƯờnG ĐẠI HỌC CÔNG NGHIỆP THÀNH PHỐ HỒ CHÍ MINH
}

\author{
NGUYẼ̃N THU HÀ, PHAN THANH HUYỀ, NGUYẼ̃N ĐìNH LUẬT \\ Trường Đại học Công nghiệp Thành phố Hồ Chí Minh; \\ hathu41@gmail.com, phanthanhhuyen@iuh.edu.vn,luatmh@gmail.com
}

Tóm tắt: Việc mắc lỗi, đặc biệt là lỗi phát âm, trong quá trình học một ngôn ngữ không phải tiếng mẹ đẻ là điều không thể tránh khỏi. Chính vì vậy một phần quan trọng của quá trình giảng dạy phát âm là phát hiện, giải thích và tìm cách giúp người học khắc phục những lỗi sai thường gặp. Không ngoài mục đích nói trên, bài nghiên cứu này được thực hiện nhằm tìm hiểu hiện tượng lỗi trong việc phát âm các phụ âm xát tiếng Anh /s/ và / - hai phụ âm tưởng chừng đơn giản nhưng lại gây khó khăn không ít cho sinh viên chuyên ngành Ngôn ngữ Anh .Bài viết tập trung xem xét việc sinh viên mắc lỗi như thế nào đối với ba trường hợp như sau: (1) vị trí phân bố âm - đầu, giữa và cuối từ; (2) các từ có chứa cả hai phụ âm /s/ và / $/$ / cũng như có tổ hợp phụ âm /s/ với các phụ âm khác; (3) những chữ viết được phát âm $/ \mathrm{s} /$ và $/ \mathrm{g} /$. Dựa vào nguyên nhân lỗi của những hiện tượng đó, nhóm nghiên cứu đề xuất giải pháp cho việc dạy và học hai phụ âm xát tiếng Anh này.

Từ khóa: lỗi, phát âm, âm xát, phụ âm, đầu từ, giữa từ, cuối từ, tổ hợp phụ âm, lần, sai, đúng, thay thế, lược bỏ, nhầm lẫn, phương thức cấu âm, vị trí câu âm.

\section{HOW ENGLISH FRICATIVES /s/ AND /f/ ARE PRODUCED BY IUH ENGLISH MAJORS}

Abstract. Mispronunciation in learning a language which is not one's mother tongue is inevitable. For
this reason, a significant part of formal instruction in pronunciation is identifying, explaining and
rectifying common mistakes. As a contribution to the accomplishment of those purposes, the paper aims
to explore the improper articulation concerning English fricatives $/ \mathrm{s} /$ and $/ \mathrm{J} /$, the consonants which seem
to be simple but as a matter of fact pose quite a challenge to English majors. Specifically, the focal point
of the paper is to examine the patterns of IUH English majors' mispronunciation with respect to (1) the
positions, including initial, middle and final, of the fricatives in a word, (2) the English words containing
both of the fricatives and those with a consonant cluster including /s/ and (3) the phonic principles behind
the spellings phonemically identified as /s/, as well as those articulated as $/ \mathrm{J} /$. Based on the findings
concerning these occurences, recommendations for the teaching and learning of these two fricative sounds
will be put forward.

Keywords. mistakes, errors, pronunciation, fricative, consonants, word-initial, word-middle, word-final, consonant clusters, turn, wrong, correct, substitute/using the wrong sound, delete/omit/leaving the sound out, manner of articulation, place of articulation.

\section{1 ĐặT VẤN ĐỀ}

Khi học một ngoại ngữ nào đó, khả năng phát âm như người bản ngữ là một tiêu chí mà cả người học lẫn người dạy ngôn ngữ đều muốn hướng đên. Trên thực tế, việc học phát âm như thế nào để đạt được hiệu quả như trên luôn là một thách thức đối với người học. Đó là vấn đề mà sinh viên chuyên ngữ cũng không phải là ngoại lệ nếu họ chỉ dừng lại ở phương pháp nghe và bắt chước - một phương pháp truyền thống vẫn thường xuyên được áp dụng để dạy và học phát âm ngoại ngữ. Vì lẽ đó, để tối ưu hóa hiệu quả của việc dạy và học phát âm tiếng Anh cho sinh viên chuyên ngữ, giáo viên giảng dạy cần nghiên cứu tìm ra phương pháp và kĩ thuật giảng dạy phù hợp, từ đó giúp sinh viên có ý thức và hứng thú với việc rèn luyện phát âm hơn.

Từ trước đừ nay, một số tác giả quan tâm đến vấn đề lỗi phát âm, phương pháp để phát âm tốt, hay thiết kế chương trình dạy phát âm tiếng Anh chuẩn cho sinh viên Việt Nam như: Khoa Anh Việt có đề tài 
nghiên cứu khoa học cấp đại học quốc gia năm 2010 với "Nghiên cứu khảo sát phát âm cho sinh viên năm nhất khoa sư phạm tiếng Anh - ĐHNN-ĐHQG HN". Đề tài tập trung đánh giá quá trình dạy và học môn phát âm trong học kỳ I, năm học 2008-2009 của sinh viên năm nhất của khoa Ngôn ngữ và Văn hóa Anh - Mỹ, trường ĐHNN - ĐHQGHN, đánh giá ưu và nhược điểm một số phương pháp dạy phát âm, đưa ra lỗi phân biệt cặp âm tối thiểu, trọng âm trong từ, trong câu trong quá trình học phát âm của người học; Nguyễn Thọ Phước Thảo với bài báo năm 2017 "Những lỗi phát âm thường gặp của sinh viên không chuyên ngữ tại trường Đại học Quảng Bình" đã phát hiện ra lỗi không phát âm âm đuôi, nhầm lẫn âm, không phát âm được tổ hợp phụ âm, việt hóa tiếng Anh, không nối âm, không nhấn trọng âm của 100 sinh viên không chuyên ngữ hệ đại học và cao đẳng tại trường Đại học Quảng Bình ; Lê Ngọc Hân trong khóa luận tốt nghiệp Đại học ngoại ngữ - Đại học quốc gia Hà Nội với đề tài "Nghiên cứu về những lỗi thường gặp của học viên người Việt khi phát âm các phụ âm tiếng Anh $/ \theta / / / \delta /, / \delta /, / 3 /, / \mathrm{d} / /, / \mathrm{t} /$ và cách khắc phục khả hữu" năm 2011 chỉ ra lỗi khi phát âm các phụ âm tiếng Anh $/ \theta /, / \delta /, / \delta / / / / /, / d ̧ 3 /, / t g /$ của 30 học sinh lớp chuyên Anh tại trường THPT chuyên Bắc Ninh; Nguyễn Thị Thơm trong luận văn thạc sĩ năm 2014 với đề tài "Nghiên cứu những lỗi phát âm thường gặp khi phát âm các phụ âm tiếng Anh của học viên tại trung tâm Anh ngữ Worldlink" đề cập đến các lỗi thay thế âm ở các phụ âm xát $/ 3 /, / / /, / 1 /$, loại bỏ âm cuối ở các phụ âm $/ \mathrm{p} /, / \mathrm{s} /, / \mathrm{f} /$ và thêm phụ âm $/ \mathrm{s} /$ của các học viên Trung tâm Anh ngữ Worldlink; Lê Thanh Hòa trong luận văn tiến sĩ "Lỗi phát âm tiếng Anh của sinh viên Việt Nam trường hợp sinh viên Đại học Đồng Nai” năm 2016 đề cập đến lỗi phát âm ở những đơn vị chiết đoạn tiếng Anh như: phụ âm đứng đầu âm tiết, phụ âm đứng cuối âm tiết, nguyên âm đôi, nguyên âm ba của 14 sinh viên ngành sư phạm tiếng Anh tại Đại học Đồng Nai được theo dõi liên tục từ năm nhất đến năm tư để tìm ra kiểu lỗi nào người học có thể khắc phục được theo thời gian học, từ đó đưa ra những giải pháp sửa lỗi phù hợp. Tuy nhiên, các nghiên cứu nêu trên chỉ dừng lại ở một số dạng lỗi phát âm tiếng Anh mà không xem xét các lỗi phát âm theo một hệ thống, và đối tượng khảo sát chủ yếu là học viên trung tâm ngoại ngữ, sinh viên không chuyên ngữ hoặc học sinh lớp chuyên, nhóm nhỏ sinh viên chuyên ngữ. nhưng chưa có một tác giả hay một đề tài nào nghiên cứu chuyên sâu về lỗi phát âm âm xát /s/ và / $/$ / tiếng Anh, hai phụ âm xát có tần suất phát âm sai cao, với qui mô khảo sát lớn tương đương 200 sinh viên chuyên ngành Ngôn ngữ Anh. Bài nghiên cứu đi sâu tìm hiểu phương thức sinh viên chuyên ngữ của Trường Đại học Công Nghiệp Tp.HCM phát âm âm xát /s/ và $/ \int /$, tìm ra các lỗi sai phổ biến và phân tích để xác định nguyên nhân dẫn đến các lỗi sai và đề xuất giải pháp khả thi cho việc dạy và học hai phụ âm tiếng Anh này.

\section{PHƯƠNG PHÁP NGHIÊN CÚU}

\subsection{Phương pháp chọn mẫu nghiên cứu}

Đối tượng nghiên cứu là lỗi phát âm âm xát /s/ và / / tiếng Anh của sinh viên năm nhất và năm ba Khoa Ngoại Ngữ, mỗi khối lớp có 100 sinh viên được chọn, với lí do như sau:

Nhiều sinh viên năm ba có biểu hiện đọc sai các âm xát này rất thường xuyên. Chúng tôi muốn kiểm tra xem sau 3 năm học, cụ thể là sau khi đã học xong môn Ngữ âm thực hành và Ngữ âm và âm vị học, khả năng phát âm các âm xát nói trên có tốt hơn so với sinh viên năm nhất hay không;

Ngoài ra, chúng tôi muốn biết các em sinh viên năm nhất có cùng khó khăn trong việc phát âm các âm xát nêu trên hay không, lỗi sai khi phát âm các phụ âm trên có phổ biến không, sai như thế nào và phổ biến ở mức độ nào để định hướng giảng dạy phát âm các âm nói trên cho sinh viên năm nhất một cách hiệu quả .

\subsection{Phương pháp thu thập dĩ liệu}

Dữ liệu nghiên cứu được thu thập dưới dạng các đoạn ghi âm trong đó sinh viên phát âm các từ và câu trong một bài kiểm tra được thiết kế sẵn. Sinh viên tham gia đọc các ngữ đoạn nêu trên và được thu âm trực tiu .

Bài kiểm tra phát âm được chia làm ba phần. Phần thứ nhất kiểm tra kĩ năng phát âm các từ riêng lẻ của sinh viên, trong đó có 22 từ có chứa âm /s/ và 20 từ chứa âm $/ \mathrm{g} /$. Trong 22 từ chứa âm $/ \mathrm{s} /$, có 8 từ có âm $/ \mathrm{s} /$ ở vị trí đầu từ, 6 từ có âm này ở giữa âm tiết và 8 từ có âm $/ \mathrm{s} /$ ở cuối âm tiết. Trong 20 từ chứa âm $/ \mathrm{J} /, 7$ từ chứa âm này ở vị trí đầu âm tiết, 6 từ chứa âm này ở vị trí giữa âm tiết và 7 từ chứa âm này ở vị trí cuối âm tiết. Phần thứ hai bao gồm 8 từ có chứa âm /s/ và / / /được sắp xếp xen kẽ ngẫu nhiên, trong đó 
có 3 từ đồng thời có cả hai phụ âm nói trên. Phần thứ ba bao gồm 20 câu với các âm tiết có chứa hai phụ âm nói trên.

Chúng tôi tiếp tục kiểm tra xem sinh viên sẽ mắc lỗi như thế nào khi phát âm các âm từ có chứa cả hai phụ âm đang xem xét, chẳng hạn conversation, solution hay supervacation. Để thuận tiện cho việc thống kê và xử lí dữ liệu, các từ có chứa cùng lúc hai âm /s/ và / $/$ / được xem như hai từ khác nhau, kết quả là có 80 từ. Và trong 80 từ trên, 19 từ có âm $/ \mathrm{s} /$ ở vị trí đầu từ, 13 từ có âm $/ \mathrm{s} /$ ở vị trí giữa từ và 16 từ có âm $/ \mathrm{s} /$ ở vị trí cuối từ. Về âm $/ \mathrm{J} /, 13$ từ có âm này ở vị trí đầu từ, 12 từ có âm này ở vị trí giữa từ và 7 từ có âm này nằm ở vị trí cuối từ. Xét trường hợp các từ có chứa tổ hợp phụ âm, phần từ tổng hợp và câu có 22 từ chứa cả hai âm $/ \mathrm{s} / \mathrm{và} / \mathrm{J} /$ và 9 từ chứa tổ hợp âm $/ \mathrm{s} /$ đứng trước hoặc theo ngay sau một phụ âm khác. Về chữ viết, 80 từ được xét cũng chứa những chữ viết thường được đọc thành âm $/ \mathrm{s} / \mathrm{như} \mathrm{s}, \mathrm{ss}, \mathrm{ce}, \mathrm{ci}, \mathrm{cy}$, $\mathrm{x}$ và âm $/ \mathrm{J} /$ như sh, ti, ci.

\subsection{Phương pháp xử lý dĩ liệu}

Trước tiên, nhóm nghiên cứu thiết kế hai mẫu phiếu chấm điểm để xác định sinh viên phát âm đúng hay sai các nội dung được kiểm tra. Mẫu phiếu thứ nhất xem xét một cách tổng quát liệu sinh viên phát âm các âm tiết đúng hay sai. Riêng phần câu nếu sinh viên đọc sai bất kì âm tiết nào có chứa âm /s/ hoặc /S/ trong câu đó thì câu này được xem như câu trả lời sai. Trường hợp sinh viên phát âm nhầm lẫn âm $/ \mathrm{s} /$ thành âm $/ g /$ hoặc ngược lại thì được tính một lỗi. Mẫu phiếu thứ hai xét từng âm tiết nằm trong danh mục các âm tiết có chứa cả hai phụ âm nói trên hoặc trong vị trí trộn lẫn vào nhau để sinh viên không mặc định được âm tiết nào có chứa âm /s/ và âm tiết nào có chứa âm $/ \mathrm{g} /$, và một phần không thể thiếu là cả các âm tiết nằm trong câu. Ngoài yếu tố đúng-sai, mẫu phiếu thứ hai này xem xét chi tiết lỗi sai ở nhiều góc độ như sau:

(1) vị trí phân bố của hai phụ âm đang được khảo sát, (2) khi phát âm các từ có chứa tổ hợp âm /s/ và / $/$ cũng như một trong hai âm này với các phụ âm khác, khả năng phát âm đúng của sinh viên có bị ảnh hưởng hay không, và mức độ ảnh hưởng có lớn không

(3) khi phát âm các từ có chữ viết có thể gây nhầm lẫn trong việc nhận biết phụ âm tương ứng như sugar, hoặc các từ có chữ viết giống tiếng Việt và phụ âm tương ứng, sinh viên có nhẫm lẫn áp dụng âm tiếng Việt vào không. Trong những trường hợp này, chúng tôi cũng tìm hiểu với những kiểu chữ viết đó, lỗi sai nào phổ biến, chẳng hạn với chữ viết là sh, sinh viên thông thường sẽ thay thế âm $/ \mathrm{g} /$ thành âm $/ \mathrm{s} /$ hay một âm nào khác, hay lược bỏ âm này hoàn toàn, hoặc có chèn thêm âm khác thường xuyên không.

Khi xem xét các vấn đề nêu trên, chúng tôi không chỉ xem xét mức độ phổ biến của các lỗi phát âm mà còn tìm hiểu cách thức phát âm sai của sinh viên, cụ thể là sinh viên có thay thế phụ âm cần đọc thành phụ âm khác trong hai âm $/ \mathrm{s} /$ hoặc $/ \mathrm{J} /$, hay thay thế bằng một phụ âm hoàn toàn khác hai âm này, sinh viên có lược bỏ phụ âm đang khảo sát hay không, và có chèn thêm một âm nào khác ở vị trí trước hoặc sau âm /s/ và / / không.

Để kiểm tra các nội dung trên, sau khi mã hóa và nhập dữ liệu vào Excel, chúng tôi sử dụng chức năng lọc thông tin của phần mềm này để tìm kiếm các từ có chứa những nội dung cần khảo sát, sau đó thống kê tần suất để phân tích, so sánh và đối chiếu thông tin để tìm ra các quy luật có thể tồn tại về lỗi phát âm của hai nhóm sinh viên.

\section{KẾT QUẢ}

Qua khảo sát, thể hiện trong bảng 1 , chúng ta có thể thấy sinh viên năm nhất đọc sai âm $/ \mathrm{s} / 25 \%$, sinh viên năm ba là $7 \%$ đọc sai âm $/ J /$ là gần $14 \%$ đối với sinh viên năm nhất và $10 \%$ đối với sinh viên năm ba. Tương tự, khi các từ có chứa hai phụ âm nói trên được sắp xếp xen kẽ và không có dấu hiệu rõ ràng âm nào đang được kiểm tra, $32 \%$ số lượt đọc sai là của sinh viên năm nhất và $27,5 \%$ thuộc về sinh viên năm thứ ba. 
Bảng 1: Kết quả thu âm tổng quát của sinh viên năm 1 và năm 3

\begin{tabular}{|c|c|c|c|c|c|c|c|c|c|c|c|c|c|c|c|c|}
\hline & \multicolumn{8}{|c|}{ từ riêng lẻ } & \multirow{2}{*}{\multicolumn{4}{|c|}{ tổng hợp cả 2 âm trong từ }} & \multirow{2}{*}{\multicolumn{4}{|c|}{$\begin{array}{c}\text { tổng hợp cả } 2 \text { âm trong } \\
\text { câu }\end{array}$}} \\
\hline & \multicolumn{4}{|c|}{$/ \mathrm{s} /$} & \multicolumn{4}{|c|}{$1 \%$} & & & & & & & & \\
\hline & \multicolumn{2}{|c|}{$\begin{array}{l}\text { Phát âm } \\
\text { đúng }\end{array}$} & \multicolumn{2}{|c|}{ Phát âm sai } & \multicolumn{2}{|c|}{$\begin{array}{l}\text { Phát âm } \\
\text { đúng }\end{array}$} & \multicolumn{2}{|c|}{ Phát âm sai } & \multicolumn{2}{|c|}{$\begin{array}{l}\text { Phát âm } \\
\text { đúng }\end{array}$} & \multicolumn{2}{|c|}{ Phát âm sai } & \multicolumn{2}{|c|}{$\begin{array}{l}\text { Phát âm } \\
\text { đúng }\end{array}$} & \multicolumn{2}{|c|}{ Phát âm sa } \\
\hline & $\begin{array}{l}\text { số } \\
\text { lần }\end{array}$ & $\begin{array}{l}\text { tỉ lệ } \\
\text { (\%) }\end{array}$ & $\begin{array}{l}\text { số } \\
\text { lần }\end{array}$ & $\begin{array}{l}\text { tỉ lê } \\
(\%)\end{array}$ & $\begin{array}{c}\text { số } \\
\text { lần }\end{array}$ & $\begin{array}{l}\text { tỉ lê } \\
(\%)\end{array}$ & $\begin{array}{l}\text { số } \\
\text { lần }\end{array}$ & $\begin{array}{l}\text { tỉ lê } \\
(\%)\end{array}$ & $\begin{array}{l}\text { số } \\
\text { lần }\end{array}$ & $\begin{array}{l}\text { tỉ lệ } \\
(\%)\end{array}$ & $\begin{array}{c}\text { số } \\
\text { lần }\end{array}$ & $\begin{array}{l}\text { tỉ lê } \\
\text { (\%) }\end{array}$ & $\begin{array}{c}\text { số } \\
\text { lần }\end{array}$ & $\begin{array}{l}\text { tỉ lệ } \\
\text { (\%) }\end{array}$ & $\begin{array}{c}\text { số } \\
\text { lần }\end{array}$ & $\begin{array}{l}\text { tîlê } \\
(\%)\end{array}$ \\
\hline $\begin{array}{c}\text { Năm } \\
1\end{array}$ & $\begin{array}{c}165 \\
2\end{array}$ & 75.1 & 548 & 24.9 & $\begin{array}{c}172 \\
8\end{array}$ & 86.4 & 272 & 13.6 & 544 & 68.0 & 256 & 32.0 & $\begin{array}{c}136 \\
4\end{array}$ & 68.2 & 636 & 31.8 \\
\hline $\begin{array}{l}\mathrm{Nă} \\
\mathrm{m} 3\end{array}$ & $\begin{array}{c}204 \\
4\end{array}$ & 92.9 & 156 & 7.1 & $\begin{array}{c}180 \\
1\end{array}$ & 90.1 & 199 & 10.0 & 580 & 72.5 & 220 & 27.5 & $\begin{array}{c}133 \\
7\end{array}$ & 66.9 & 663 & 33.2 \\
\hline
\end{tabular}

Hai nhóm sinh viên phát âm sai nhiều nhất khi phát âm các từ có chứa cả hai phụ âm hoặc các từ có chứa một trong hai âm được trộn lẫn vào nhau theo nhóm và theo từng câu. Theo bảng 1 , số lần phát âm sai của sinh viên năm nhất khi phát âm xát $/ \mathrm{s} / \mathrm{và} / \mathrm{J} /$ trong cả hai trường hợp đều xấp xỉ $32 \%$. Số lần phát âm sai của sinh viên năm ba khi phát âm $/ \mathrm{s} /$ trong các từ tổng hợp chiểm tương đương $28 \%$ và trong câu là khoảng $33 \%$ - đây cũng là trường hợp duy nhất lỗi sai của sinh viên năm ba chiếm tỉ lệ cao hơn so với sinh viên năm nhất.

Khi phát âm các từ có chứa âm xát /s/ và / / trong dãy âm tiết tổng hợp và trong câu, sinh viên không có bất kì dấu hiệu nào từ đề bài kiểm tra và phải tự nhận diện từ nào hoặc âm tiết nào có chứa âm xát /s/ và âm $/ \mathrm{g} /$ để đọc. Lúc này lỗi sai của sinh viên có thể do hai nguyên nhân như sau. Thứ nhất, các sinh viên không kiểm soát được các bộ phận tham gia vào quá trình cấu âm, nói cách khác là vị trí cấu âm khi phải liên tục chuyển đổi các vị trí cấu âm hay luân phiên sử dụng các bộ phận cấu âm khác nhau. Thứ hai, các sinh viên nhầm lẫn giữa chữ viết, thậm chí là chữ viết tiếng Việt với phụ âm được sử dụng, dẫn đến việc áp dụng sai nguyên tắc phát âm của tiếng Anh hoặc sử dụng nguyên tắc phát âm của tiếng Việt dựa trên chữ viết vào việc phát âm các từ và âm tiết tiếng Anh. Vì vậy bài viết tiếp tục đi sâu xử lí và phân tích dữ liệu phát âm của sinh viên liên quan đến lỗi sai khi xét về vị trí của các phụ âm đang xem xét và cách thức các em mắc lỗi (thay thế âm, lược bỏ âm, nhầm lẫn chữ viết với phụ âm) để tìm hiểu liệu các lỗi sai đó có tuân theo quy luật cụ thể nào không.

\subsection{Lỗi phát âm sai liên quan đến vị trí phân bố âm}

Các lỗi phát âm sai của hai nhóm sinh viên được thống kê dựa trên tỉ lệ của các lỗi sai so với tổng số lần phát âm sai của từng trường hợp và tóm tắt trong bảng dưới đây.

Bảng 2: Tỉ lệ của các lỗi phổ biến khi phát âm /s/ và $/ \mathrm{g} /$ trong từ chứa cả hai âm và trong câu

\begin{tabular}{|l|r|r|}
\hline \multirow{2}{*}{ Phương thức phát âm } & \multicolumn{2}{|c|}{ Tỉ lệ lỗi (\%) } \\
\cline { 2 - 3 } & Năm 1 & Năm 3 \\
\hline Thay thế âm /s/ thành âm /g/ & 30.5 & 59.7 \\
\hline Lược bỏ âm /s/ & 65.3 & 31.4 \\
\hline Thay thế âm / / thành âm /s/ & 80.9 & 84.4 \\
\hline Lược bỏ âm / $/$ & 13.9 & 4.3 \\
\hline
\end{tabular}

Bảng thống kê cho thấy đối với sinh viên năm ba, với tỉ lệ lược bỏ âm /s/ chiếm tương đương $30 \%$ và âm $/ \mathrm{J} /$ tương đương $4 \%$, lỗi lược bỏ hai phụ âm xát có tỉ lệ thấp hơn một nửa so với sinh viên năm nhất, với tỉ lệ mắc lỗi lược bỏ âm /s/ và / / lần lượt vào khoảng $65 \%$ và $14 \%$. Tuy nhiên, việc nhầm lấn hai phụ âm này với nhau lại xảy ra thường xuyên hơn ở nhóm sinh viên năm 3, với tỉ lệ vào khoảng $60 \%$ đối với âm $/ \mathrm{s} /$, gấp đôi so với sinh viên năm nhất và gần $85 \%$ đối âm $/ \mathrm{J} /$. Tỉ lệ trên đối với sinh viên năm nhất lần lượt vào khoảng $30 \%$ và $80 \%$.

Về vị trí phân bố, như Bảng 7 minh họa dưới đây, vị trí đầu tù của cả hai phu âm xát là vị trí có tỉ lệ nhầm lẫn giữa hai phụ âm xát cao nhất đối với cả hai nhóm sinh viên, với lỗi thay thế âm /s/ thành 
âm $/ \int /$ chiếm xấp xỉ 93\% và ngược lại lỗi thay thế âm / $/$ thành âm /s/ chiếm tương đương $96 \%$ đối với sinh viên năm nhất. Tỉ lệ trên đối với sinh viên năm ba ở vào khoảng $89 \%$ và $93 \%$.

Bảng 3: Tỉ lệ của các lỗi phổ biến trong phương thức phát âm $/ \mathrm{s} /$ và $/ \mathrm{g} /$ theo vị trí phân bố

\begin{tabular}{|c|c|c|c|c|c|c|}
\hline \multirow{2}{*}{$\begin{array}{c}\text { Vị trí âm/ } \\
\text { Phương thức phát âm }\end{array}$} & \multicolumn{2}{|c|}{ Đầu từ $(\%)$} & \multicolumn{2}{|c|}{ Giữa từ $(\%)$} & \multicolumn{2}{|c|}{ Cuối từ $(\%)$} \\
\hline & Năm 1 & Năm 3 & Năm 1 & Năm 3 & Năm 1 & Năm 3 \\
\hline Thay thế âm /s/ thành âm / $/$ & 92.6 & 88.8 & 35.7 & 67.5 & 3.3 & 12.3 \\
\hline Lược bỏ âm /s/ & 7.4 & 5.2 & 55.7 & 22.2 & 93.9 & 76.9 \\
\hline Thay thế âm / $/$ thành âm /s/ & 95.8 & 92.9 & 86.4 & 73.1 & 63.3 & 84.4 \\
\hline Lược bỏ âm / $/$ & 0.7 & 0.0 & 2.9 & 1.4 & 34.5 & 12.1 \\
\hline
\end{tabular}

Ơ vị trí giữa tù̀, việc nhầm lẫn hai phụ âm xát còn chiếm tỉ lệ khá cao ở cả hai nhóm sinh viên. Nhóm sinh viên năm nhất có số lần thay thế phụ âm /s/ thành $/ \mathrm{S} /$ chiếm gần $36 \%$ tổng số lần phát âm sai và ngược lại âm $/ \mathrm{S} /$ thành âm $/ \mathrm{s} / \mathrm{c}$ chiếm đến trên $86 \%$. Bên cạnh đó, sinh viên năm nhất còn mắc phải lỗi lược bỏ âm $/ \mathrm{s} /$ khá thường xuyên, chiếm gần $56 \%$. Điều đặc biệt là lỗi thay thế âm $/ \mathrm{s} /$ thành âm $/ \mathrm{J} /$ ở sinh viên năm ba có tỉ lệ gần gấp đôi so với sinh viên năm nhất, cụ thể là vào khoảng $68 \%$, ngoài ra lỗi thay thế âm $/ \mathrm{S} /$ thành âm $/ \mathrm{s} /$ cũng chiếm đến $73 \%$.

Vị trí cuối tù̀ cũng cho thấy sự nhất quán về cách thức phát âm sai của hai nhóm sinh viên mặc dù khuynh hướng phạm lô̂i đối với âm /s/ và âm / $/$ có sự khác biệt. Đối voói âm $/ \mathbf{s} /$, sinh viên có khuynh hướng không phát âm phụ âm này ở vị trí cuối tù̀, với tỉ lệ gần $94 \%$ đối với sinh viên năm nhất và xấp xỉ $77 \%$ đối với sinh viên năm ba. Ngược lại, khi phát âm phụ âm /fl ở vị trí cuối tù̀, sinh viên thường xuyên thay thế âm này thành âm /s/. Tỉ lệ mắc lỗi như thế chiếm trên $63 \%$ ở sinh viên năm nhất, còn sinh viên năm ba thậm chí còn lên đến trên $84 \%$. Ngoài ra cũng cần lưu ý rằng sinh viên năm nhất còn loại bỏ âm /g/ ở vị trí cuối từ khá thường xuyên, với tỉ lệ lỗi chiếm gần $35 \%$.

Nhìn chung, lỗi phát âm phổ biến nhất là nhầm lẫn hai phụ âm xát với nhau, trong đó sinh viên năm ba dù đã học môn Ngữ âm thực hành nhưng lại mắc phải lỗi này (mistake) còn thường xuyên hơn sinh viên năm nhất. Vốn chưa học qua học phần $\mathrm{Ngữ} \mathrm{âm} \mathrm{thực} \mathrm{hành,} \mathrm{sinh} \mathrm{viên} \mathrm{năm} \mathrm{nhất} \mathrm{còn} \mathrm{mắc} \mathrm{lỗi} \mathrm{(error)}$ lược bỏ âm $/ \mathrm{s} /$ khá thường xuyên. Ở vị trí đầu từ và giữa từ, lỗi phát âm phổ biến là nhầm lẫn hai phụ âm xát, riêng sinh viên năm nhất còn mắc phải lỗi lược bỏ âm /s/ ở vị trí giữa từ. Ở vị trí cuối từ, sinh viên có khuynh hướng không phát âm âm /s/ và thay thế âm /g/ thành âm /s/, ngoài ra còn có một loại lỗi khác mà sinh viên năm nhất mắc phải dù ít phổ biến hơn là lược bỏ âm $/ \mathrm{g} /$.

\subsection{Lỗi phát âm sai liên quan đến tổ hợp âm}

\subsubsection{Hai âm /s/và /f/ cùng xuất hiện trong một tù}

Khi hai phụ âm xát nói trên cùng xuất hiện trong một từ, việc phát âm đúng hai âm này có thể sẽ khó khăn hơn. Và kết quả phần thu âm đã cho thấy điều đó rất rõ ràng. Khi xét hiệu quả phát âm âm $/ \mathrm{s} /, \sinh$ viên năm thứ nhất mắc lỗi trên $27 \%$ lần phát âm. Tỉ lệ này đã giảm đáng kể ở sinh viên năm thứ ba, với $19 \%$ lần phát âm sai âm $/ \mathrm{s} /$. Và một lần nữa, hai lỗi phổ biến nhất vẫn là thay thế âm $/ \mathrm{s} /$ thành âm $/ \mathrm{S} /$ và bỏ qua không phát âm âm $/ \mathrm{s} /$. Kết quả này cũng cho thấy sự nhất quán với qui luật phát âm sai âm $/ \mathrm{s} /$ nói chung như đã được phân tích ở trên. Cụ thể là sinh viên năm thứ ba mắc lỗi thay thế âm $/ \mathrm{s} /$ thành âm $/ \mathrm{J} /$ trong 198 lần phát âm, trong khi số lần mắc lỗi này của sinh viên năm thứ nhất là 109 . Điều này cho thấy sự nhầm lẫn giữa hai phụ âm xát nói trên sau khi sinh viên được học phát âm hai phụ âm này rất đáng quan tâm. Ngược lại, xét về lỗi loại bỏ âm thì sinh viên năm ba thể hiện sự cải thiện đáng kể khi chỉ mắc phải lỗi này ở 76 lần phát âm. Trong khi đó, sinh viên năm thứ nhất chưa trải qua quá trình đào tạo về phát âm tiếng Anh đã có 269 lần không phát âm phụ âm $/ \mathrm{s} /$.

Bảng 4: Kết quả phát âm âm /s/ trong các từ có chứa cả hai âm 


\begin{tabular}{|c|c|c|c|c|c|}
\hline \multirow{2}{*}{\multicolumn{2}{|c|}{ Phương thức phát âm }} & \multicolumn{4}{|c|}{$/ \mathrm{s} /$} \\
\hline & & \multicolumn{2}{|c|}{ Năm 1} & \multicolumn{2}{|c|}{ Năm 3} \\
\hline \multicolumn{2}{|r|}{ Đúng } & 1100 & $73 \%$ & 1211 & $81 \%$ \\
\hline \multirow{6}{*}{$\begin{array}{c}\text { Thay thế } \\
\text { âm }\end{array}$} & $\mid \mathrm{g} /$ & 109 & \multirow{10}{*}{$27 \%$} & 198 & \multirow{10}{*}{$19 \%$} \\
\hline & $|\mathrm{z}|$ & 10 & & 7 & \\
\hline & không rõ /s/ hay / $/$ / & & & 3 & \\
\hline & $/ \mathrm{v} /$ & 6 & & 2 & \\
\hline & $/ \mathrm{t} /$ & 2 & & 1 & \\
\hline & $/ \mathrm{n} /$ & 2 & & & \\
\hline & $/ \mathrm{r} /$ & 1 & & & \\
\hline \multicolumn{2}{|c|}{ Lược bỏ âm } & 269 & & 76 & \\
\hline \multirow[t]{2}{*}{ Thêm âm } & $/ \mathrm{t} /$ & 1 & & 1 & \\
\hline & $\mid \mathrm{S} /$ & & & 1 & \\
\hline \multicolumn{2}{|c|}{ Tổng số lần phát âm } & 1500 & $100 \%$ & 1500 & $100 \%$ \\
\hline
\end{tabular}

Xét về phụ âm trên nướu răng $/ \mathrm{J} /$, như được trình bày trong bảng thống kê số 7 dưới đây, khuynh hướng mắc lỗi của sinh viên thuộc hai nhóm lớp thể hiện khá hợp lí, với tỉ lệ mắc lỗi của sinh viên năm nhất $(16 \%)$ cao hơn mức $9 \%$ của sinh viên năm ba. Đối với phụ âm này, cả hai nhóm sinh viên đều hạn chế được việc bỏ qua không phát âm phụ âm này ở mức tối thiểu, vì sinh viên năm thứ ba không mắc lỗi này khi phát âm các từ có chứa cả hai phụ âm xát đang xem xét, còn sinh viên năm nhất chỉ có 2 lần loại bỏ âm này. Tuy nhiên vẫn có 82 và 45 lần phát âm $/ \mathrm{J} /$ thành âm $/ \mathrm{s} /$ ở sinh viên năm nhất và năm ba. Như vậy khi phải phát âm phụ âm xát /S/ trong một từ có cả hai phụ âm nói trên, sinh viên năm thứ ba có sự cải thiện khá tốt, hạn chế được việc lẫn lộn phụ âm này với âm nướu răng /s/.

Bảng 5: Kết quả phát âm âm $/ \mathrm{J} /$ trong các từ có chứa cả hai âm

\begin{tabular}{|c|c|c|c|c|c|}
\hline \multirow{2}{*}{\multicolumn{2}{|c|}{ Phương thức phát âm }} & \multicolumn{4}{|c|}{$/ \mathrm{d} /$} \\
\hline & & \multicolumn{2}{|c|}{ Năm 1} & \multicolumn{2}{|c|}{ Năm 3} \\
\hline \multicolumn{2}{|c|}{ Đúng } & 506 & $84 \%$ & 545 & $91 \%$ \\
\hline \multirow{3}{*}{$\begin{array}{l}\text { Thay } \\
\text { thế }\end{array}$} & $/ \mathrm{s} /$ & 82 & \multirow{9}{*}{$16 \%$} & 45 & \multirow{9}{*}{$9 \%$} \\
\hline & $|z|$ & 7 & & & \\
\hline & \multicolumn{2}{|c|}{ không rõ /s/ hay / / / } & & 3 & \\
\hline \multirow[t]{3}{*}{ âm } & 121 & & & 5 & \\
\hline & 11 & 1 & & & \\
\hline & $/ \mathrm{t} /$ & 1 & & 1 & \\
\hline \multicolumn{2}{|c|}{ Lược bỏ âm } & 2 & & & \\
\hline \multirow{2}{*}{ Thêm âm } & $/ \mathrm{n} /$ & 1 & & & \\
\hline & $/ \mathrm{s} /$ & & & 1 & \\
\hline \multicolumn{2}{|c|}{ Tổng số lần phát âm } & 600 & $100 \%$ & 600 & $100 \%$ \\
\hline
\end{tabular}

Từ các dữ liệu nêu trên, chúng tôi xét tỉ lệ của những lỗi phổ biến nhất so với tổng số lần mắc lỗi và tóm tắt trong bảng dưới đây.

Bảng 6: Tỉ lệ lỗi phát âm khi âm /s/ và / $/$ / cùng xuất hiện trong từ

\begin{tabular}{|c|c|c|}
\hline \multirow{2}{*}{ Phương thức phát âm âm } & \multicolumn{2}{|c|}{ Tỉ lệ \% } \\
\cline { 2 - 3 } & Năm 1 & Năm 3 \\
\hline Thay thế âm /s/ thành âm/g/ & 27.3 & 68.5 \\
\hline Lược bỏ âm /s/ & 67.3 & 26.3 \\
\hline Thay thế âm / / thành âm/s/ & 87.2 & 81.8 \\
\hline
\end{tabular}


Theo các số liệu trong Bảng 6, lỗi phát âm phổ biến khi hai phụ âm xát cùng xuất hiện trong một từ vẫn là lỗi nhầm lẫn hai phụ âm xát với nhau, đặc biệt là việc nhầm lẫn âm $/ \mathrm{g} /$ thành âm $/ \mathrm{s} /$, chiếm đến trên $80 \%$ các lần phát âm sai của cả hai nhóm sinh viên. Lỗi thay thế âm / $/ \mathrm{s} /$ thành âm $/ \mathrm{g} /$ thậm chí còn thường xuyên xảy ra hơn ở nhóm sinh viên năm ba, với số lần mắc lỗi chiếm xấp xỉ $70 \%$, so với sinh viên năm nhất chỉ tương đương $27 \%$.

\subsubsection{Tổ hợp âm /s/và một phu âm khác}

Vì phụ âm $/ \mathrm{S} /$, như các tác giả Celce-Murcia, M., Brinton, D.M và Goodwin J.M. (trang 81 ) đã đề cập, không bao giờ xuất hiện trong tổ hợp âm, bài viết xem xét bốn tổ hợp âm phổ biến liên quan đến âm $/ \mathrm{s} /$ như đã trình bày trong bảng thống kê só 8 dưới đây.

Bảng 7: Kết quả phát âm âm /s/ trong các từ có chứa tổ hợp âm

\begin{tabular}{|c|c|c|c|c|c|c|c|c|c|c|c|c|}
\hline \multirow{2}{*}{\multicolumn{2}{|c|}{$\begin{array}{c}\text { Tổ hợp âm } \\
\text { Phương thức phát âm }\end{array}$}} & \multicolumn{3}{|c|}{$/ \mathrm{ks} /$} & \multicolumn{3}{|c|}{ /sk/ } & \multicolumn{3}{|c|}{$/ \mathrm{st} /$} & \multicolumn{2}{|c|}{$/$ str/ } \\
\hline & & Năm 1 & Tỉ lệ & Năm 3 & Năm 1 & Tỉ lệ & Năm 3 & Năm 1 & Tỉ lệ & Năm 3 & Năm 1 & Năm 3 \\
\hline \multicolumn{2}{|r|}{ đúng } & 147 & $74 \%$ & 179 & 230 & $77 \%$ & 267 & 347 & $87 \%$ & 378 & 100 & 94 \\
\hline \multirow{3}{*}{$\begin{array}{l}\text { Thay } \\
\text { thế } \\
\text { âm }\end{array}$} & $/ \mathrm{g} /$ & & \multirow{6}{*}{$26 \%$} & 4 & 3 & \multirow{6}{*}{$23 \%$} & 9 & 2 & \multirow{6}{*}{$13 \%$} & 4 & & 1 \\
\hline & $|z|$ & 1 & & & & & 2 & & & & & \\
\hline & không rõ /s/ hay /S/ & & & & & & 2 & & & 1 & & \\
\hline \multicolumn{2}{|c|}{ Lược bỏ âm } & 52 & & 16 & 67 & & 19 & 51 & & 17 & & 5 \\
\hline \multirow{2}{*}{$\begin{array}{l}\text { Thêm } \\
\text { âm }\end{array}$} & $/ \mathrm{t} /$ & & & 1 & & & & & & & & \\
\hline & $/ \mathrm{d} /$ & & & & & & 1 & & & & & \\
\hline \multicolumn{2}{|c|}{ Tổng số lần phát âm } & 200 & $100 \%$ & 200 & 300 & $100 \%$ & 300 & 400 & $100 \%$ & 400 & 100 & 100 \\
\hline
\end{tabular}

Số liệu từ bảng 8 cho thấy tổ hợp âm/str/ không gây khó khăn cho sinh viên. Tổ hợp âm gây trở ngại nhiều nhất là $/ \mathrm{ks} /$, khiến cho $26 \%$ lần phát âm của sinh viên năm nhất đối với tổ hợp này thiếu chính xác. Tỉ lệ sai là $23 \%$ khi sinh viên năm nhất phát âm tổ hợp phụ âm /sk/. Mặc dù tổ hợp /st/ có tỉ lệ phát âm đúng khá cao, lỗi phổ biến của sinh viên khi phát âm tổ hợp này là loại bỏ âm $/ \mathrm{s} /$, với 51 lần phát âm có hiện tượng này. Cần lưu ý là đối với tổ hợp /ks/, sinh viên sẽ không phát âm phụ âm $/ \mathrm{k} /$, còn đối với tổ hợp /sk/ và /st/, sinh viên sẽ có thói quen bỏ qua âm /s/. So với sinh viên năm thứ nhất, sinh viên năm thứ ba không gặp khó khăn nhiều đối với các tổ hợp âm.

Như vậy, hiện tượng bỏ qua không phát âm /s/ và /k/ là hiện tượng phổ biến đối với sinh viên năm thứ nhất tại thời điểm chưa được học môn Ngữ âm thực hành, khi phát âm các tổ hợp /ks/ và /sk/.

\subsection{Lỗi phát âm liên quan đến chĩ viết}

Để so sánh mức độ phổ biến của các loại lỗi khi phát âm phụ âm/s/ và / $/$, tỉ lệ của các lỗi trên tổng số lần phát âm sai của sinh viên được tóm tắt trong bảng thống kê dưới đây.

Đối với chữ viết $c i$ và $s s$, vốn là các chữ viết có thể được phát âm $/ \mathrm{s} /$ hoặc $/ \mathrm{J} /$, lỗi phổ biến là nhầm lẫn hai phụ âm này với nhau, với tỉ lệ thấp nhất cũng đã xấp xỉ $50 \%$ và cao nhất là gần $93 \%$. Ngoài ra sinh viên năm nhất còn lược bỏ âm $/ \mathrm{s} /$ (chiếm gần $32 \%$ tổng số lỗi), nhầm âm $/ \mathrm{g} /$ thành âm $/ \mathrm{k} / \mathrm{khi}$ phát âm chữ $c i(34.6 \%)$ và sinh viên năm ba thì thay thế âm $/ \mathrm{J} /$ thành âm $/ 2 /$ khi phát âm chữ $s s$ (gần $30 \%$ ).

Bảng 8: Tỉ lệ phát âm sai âm/s/ và / $/$ xét về mối quan hệ với chữ viết

\begin{tabular}{|c|c|c|c|c|c|c|c|c|c|c|c|c|c|c|c|c|c|}
\hline \multirow{3}{*}{\multicolumn{2}{|c|}{$\begin{array}{l}\text { Phương thức phát } \\
\text { âm }\end{array}$}} & \multicolumn{16}{|c|}{ Tỉ lệ phát âm sai âm /s/ hoặc / / / trên tổng số lỗi (\%) } \\
\hline & & \multicolumn{2}{|c|}{$\mathrm{ci}$} & \multicolumn{2}{|c|}{ ss } & \multicolumn{2}{|c|}{ cy } & \multicolumn{2}{|c|}{$\mathrm{s}$} & \multicolumn{2}{|c|}{ ce } & \multicolumn{2}{|c|}{$\mathrm{x}$} & \multicolumn{2}{|c|}{ sh } & \multicolumn{2}{|c|}{$\mathrm{ti}$} \\
\hline & & Năm 1 & Năm 3 & Năm 1 & Năm 3 & Năm 1 & Năm 3 & Năm 1 & Năm 3 & Năm 1 & Năm 3 & Năm 1 & Năm 3 & Năm 1 & $\begin{array}{c}\text { Năm } \\
3\end{array}$ & Năm 1 & Năm 3 \\
\hline \multicolumn{2}{|c|}{$\begin{array}{c}\text { Thay thế âm } / \mathrm{s} / \text { bằng } \\
\text { âm } / \mathrm{g} /\end{array}$} & 61.5 & 72.1 & 65.9 & 74 & 100 & 87.5 & 44.6 & 67.4 & 1.8 & 17.6 & 0 & 19 & & & & \\
\hline \multicolumn{2}{|c|}{ Lược bỏ âm /s/ } & 0 & 2.3 & 31.8 & 20 & 0 & 0 & 50.9 & 24.7 & 95.2 & 73.5 & 98.1 & 76.2 & & & & \\
\hline \multirow{3}{*}{$\begin{array}{c}\text { Thay thế } \\
\text { âm } / \delta / \\
\text { bằng }\end{array}$} & $/ \mathrm{s} /$ & 63.5 & 60.9 & 92.6 & 44.8 & & & & & & & & & 78.7 & 89.5 & 92.3 & 89.6 \\
\hline & $/ \mathrm{k} /$ & 34.6 & 17.4 & 0 & 0 & & & & & & & & & 0.2 & 0 & 0 & 0 \\
\hline & 121 & 0 & 0 & 0 & 29.9 & & & & & & & & & 0.2 & $\overline{0}$ & 0 & 4.5 \\
\hline
\end{tabular}

Trong các chữ viết còn lại có liên quan đến âm /s/, $c y$ và $s$ là những chữ viết khiến cho sinh viên cả hai nhóm thường xuyên nhầm lẫn âm /s/ thành âm $/ \mathrm{J} /$, thậm chí đối với chữ viết $c y$, sinh viên năm nhất luôn đọc nhầm thành âm $/ \mathrm{S} / \mathrm{khi}$ mắc lỗi, với tỉ lệ lỗi là $100 \%$. Đối với chữ viết $s$, vốn là chữ viết rất thường xuyên được phát âm $/ \mathrm{s} /$, nhưng sinh viên năm ba lại phát âm thành âm $/ \mathrm{J} /$, với tỉ lệ trên $67 \%$, cao 
hơn nhiều so với sinh viên năm nhất với tỉ lệ gần $45 \%$, và khoảng $51 \%$ số lỗi còn lại là do sinh viên năm nhất lược bỏ âm $/ \mathrm{s} /$.

Đối với chữ viết $c e$ và $x$, sinh viên hai nhóm có khuynh hướng lược bỏ âm /s/ với tỉ lệ mắc lỗi khá cao, cụ thể là vào khoảng $95 \%$ với chữ $c e$ và $98 \%$ với chữ $x$ ở sinh viên năm nhất. Tỉ lệ này ở nhóm sinh viên năm ba lần lượt là khoảng $74 \%$ và $76 \%$.

Trong số các chữ viết còn lại liên quan đến âm $/ \mathrm{J} /$, bao gồm $s h$ và $t i$, lỗi phát âm chiếm số lượng nhiều vẫn là nhầm lẫn âm $/ \mathrm{J} /$ thành âm $/ \mathrm{s} /$, với tỉ lệ lỗi chiếm từ $80 \%$ đến trên $92 \%$. Đặc biệt là với tỉ lệ gần $90 \%$, sinh viên năm ba còn mắc lỗi nhầm lẫn này cao hơn cả sinh viên năm nhất (tương đương $79 \%$ ) khi phát âm chữ viết sh.

Nhìn chung, có thể thấy chũ viết ci và ss là hai kiểu chũ̃ viết vừa có thể được phát âm $/ \mathrm{s} /$ vù̀a có thể được phát âm là /J/ nên việc sinh viên nhầm lẫn âm /s/thành âm //là điều dễ hiểu. Trong khi sinh viên thuò̀ng xuyên nhầm lẫn âm /s/thành âm /J/ khi phát âm các chü viết cy và s cũng nhu thay thế âm $/ \int /$ thành âm $/ \mathrm{s} /$ khi phát âm chũ sh và ti, khuynh huớng chung khi phát âm chũ viết ce và $x$ lại là luọc bỏ âm $/ \mathrm{s} /$.

\section{KẾT LUẬN}

Từ kết quả nghiên cứu đã trình bày ở trên, chúng tôi đưa ra một số đề xuất với nhà trường, giảng viên và sinh viên như sau.

Trước hết, nhà trường cần có cơ chế tuyển dụng giáo viên bản ngữ chuyên ngành Ngôn ngữ Anh giảng dạy các lớp chuyên ngữ để giúp sinh viên có cơ hội được tiếp xúc với tiếng Anh chuẩn, không đơn giản chỉ phục vụ cho việc học theo hình thức bắt chước mà giáo viên người bản ngữ là minh chứng cụ thể cho việc hướng dẫn đúng khẩu hình, vị trí cấu âm ...từ đó cải thiện kỹ năng phát âm tiến gần hơn với phát âm của người bản ngữ - một ưu thế khi tham gia vào thị trường lao động, nhất là trong ngành giảng dạy tiếng Anh.

Về phía giảng viên, cần lưu ý rằng việc nhầm lẫn hai phụ âm xát diễn ra rất thường xuyên, ngay cả khi sinh viên đã học phát âm hai phụ âm này, vì vậy khi giúp sinh viên luyện tập phát âm các âm /s/, / $/$ trong các từ có chứa cả hai phụ âm và trong câu, giảng viên cần hướng dẫn chi tiết cho sinh viên về vị trí cấu âm và phương thức cấu âm của hai phụ âm xát $/ \mathrm{s} /$ và $/ \mathrm{g} /$, trong đó cần chú ý nêu rõ các nét $\boldsymbol{k} h \boldsymbol{u}$ biệt của hai phụ âm này để giúp sinh viên không bị lẫn lộn. Cụ thể là âm /s/ là âm nướu răng, vì vậy sinh viên phải đặt đầu lưỡi tiếp xúc với phần nướu ở phía sau hàm răng trên, còn âm / $/$ là phụ âm trên nướu răng nên mặt lưỡi phải đặt cao hơn nướu răng, tiến gần về phía ngạc cứng. Một đặc điểm quan trọng nữa mà các tác giả Avery và Ehrlich (1995:16), O’Connor (1986:35) và Roach (2014:42) đã đề cập là âm / $/$ / là phụ âm đòi hỏi có sự tròn môi, vì vậy để giúp sinh viên có thể nhận thức được điều này giảng viên có thể yêu cầu sinh viên mang theo một chiếc gương nhỏ và quan sát điều chỉnh hình dáng môi cho chính xác. Đây chính là kĩ thuật giảng dạy phát âm mà tác giả Lujan (2014:2.3) và Ashton và Shepherd (2013:12) đã đề xuất nhằm giúp sinh viên nhìn thấy được sự di chuyển của các cơ quan cấu âm mà điển hình là môi.

Về vị trí phân bố âm, khi huấn luyện sinh viên phát âm các từ riêng lẻ, giảng viên cần chú ý đến vị trí đầu và giữa từ của âm $/ \mathrm{s} /$ cũng như vị trí giữa và cuối từ của âm $/ \mathrm{J} /$. Khi giúp sinh viên luyện tập phát âm các âm $/ \mathrm{s} /$ và $/ \mathrm{S} /$ trong các từ có chứa cả hai phụ âm cũng như trong câu, giảng viên cần lưu ý rằng khi phát âm hai phụ âm xát ở vị trí đầu từ và giữa từ, sinh viên có khuynh hướng nhầm lẫn hai âm này với nhau, và sinh viên năm nhất sẽ thường xuyên mắc phải lỗi lược bỏ âm /s/ ở vị trí giữa từ. Ở vị trí cuối từ, sinh viên có thói quen không phát âm âm $/ \mathrm{s} /$ và nhầm âm $/ \mathrm{J} /$ thành âm $/ \mathrm{s} /$.

Khi dạy phát âm các từ có chứa cả hai phụ âm xát nói trên, giảng viên cần nhớ rằng lỗi nhầm lẫn hai phụ âm xát với nhau vẫn là lỗi phổ biến, đặc biệt là việc nhầm lẫn âm $/ \mathrm{g} /$ thành âm $/ \mathrm{s} / \mathrm{của}$ sinh viên năm nhất khi chưa học phát âm tiếng Anh. Bên cạnh đó, lỗi thay thế âm $/ \mathrm{s} /$ thành âm $/ \mathrm{g} /$ thậm chí còn thường xuyên xảy ra hơn sau khi sinh viện học xong bài học phát âm hai âm xát này. Ngoài ra, tổ hợp / ks/ và /sk/ là hai tổ hợp phụ âm gây khó khăn cho sinh viên học phát âm lần đầu. Để khắc phục tình trạng không phát âm âm /s/ trong hai tổ hợp này, giảng viên có thể áp dụng phương pháp loại bỏ dần âm tiết (resyllabification) mà các nhà nghiên cứu Celce-Murcia, Brinton và Goodwin (1996:83) đã đề xuất để giúp sinh viên phát âm đúng các tổ hợp này. Chẳng hạn, đầu tiên giảng viên có thể yêu cầu các em phát âm cụm từ sick sister, sau khi sinh viên đọc lớn cụm từ này vài lần, giảng viên bắt đầu lược bỏ âm 
tiết ter của từ sister và yêu cầu sinh viên tiếp tục tập phát âm lại cụm âm tiết mới. Sau đó, giáng viên tiếp tục xóa bỏ chữ viết is và yêu cầu sinh viên tiếp tục đọc phần còn lại.

Khi hướng dẫn sinh viên nhận biết hai phụ âm xát thông qua chữ viết, giảng viên nên yêu cầu sinh viên tra từ điển những từ có chứa chữ viết $c i$ và $s s$ và ghi chép lại kí hiệu phiên âm, vì đây là những kiểu chữ viết vừa có thể được phát âm $/ \mathrm{s} /$ vừa có thể được phát âm là $/ \mathrm{S} /$ nên rất dễ nhầm lẫn hai phụ âm này với nhau. Giảng viên cũng cần lưu ý sinh viên rằng chữ viết $c y, s$ là chữ viết khá đặc trưng của âm $/ \mathrm{s} /$, còn $s h$ và $t i$ là chữ viết tiêu biểu của âm $/ \mathrm{J} /$, đồng thời nhắc nhở sinh viên không bỏ qua phụ âm $/ \mathrm{s} /$ khi phát âm chữ viết $c e$ và $x$.

Về phía sinh viên, một số lỗi phát âm có thể đã thành thói quen lâu năm, nên khi luyện tập phụ âm xát /s/ và / $/$ / nói riêng và các phụ âm khác nói chung, sinh viên cần tìm hiểu kĩ vị trí và phương thức cấu âm của các âm đó, từ đó tìm ra điểm khác biệt cơ bản của âm đó so với các âm khác trong tiếng Anh và thậm chí có thể đối chiếu với âm /s/, /ş/ tiếng Việt để tránh sự nhầm lẫn, sinh viên cần đối chiếu xem mình đã phát âm giống người bản ngữ chưa để tự khắc phục các lỗi phát âm, chú ý các từ có chữ viết tương tự để mở rộng sửa lỗi phát âm cuả mình một cách có hệ thống hơn.

\section{TÀI LIÊUU THAM KHẢO}

[1] Ashton, H. \& Shepherd, S. (2013), Work on Your Accent - Clearer Pronunciation for Better Communication, HarperCollins Publishers Limited.

[2] Avery, P. and Ehrlich, S. (1995), Teaching American English Pronunciation, Oxford University Press.

[3] Celce-Murcia, M., Brinton, D.M. and Goodwin, J. (1996), Teaching Pronunciation - A Reference for Teachers of English to Speakers of Other Languages, Cambridge University Press.

[4] Đoàn Thiện Thuật (1977), Sách ngữ âm tiếng việt, NXB ĐH \& THCN, Hà Nội

[5] Lujan, B.A. (2004), The American Accent Guide - A comprehensive Course on the Sound System of American English, Lingual Arts

[6] Mai Ngọc Chừ (1997), Cơ sở ngôn ngữ học và tiếng Việt, NXB Giáo dục, Hà Nội

[7] Nguyễn Thiện Giáp (2008), Giáo trình ngôn ngữ học, NXB ĐHQG HN

[8] O’Connor,J.D. (1986), Better English Pronunciation, Cambridge University Press

[9] O’Connor,J.D. (1991), Sounds English, Cambridge University Press

[10] Poedjosoedarmo, G. (2004), Teaching Pronunciation - Why, What, When, and How, SEAMEO Regional Language Centre

[11] Roach, P. (2014), English Phonetics and Phonology, Cambridge University Press.

Ngày nhận bài: 13/03/2018 Ngày chấp nhận đăng: 09/09/2018 